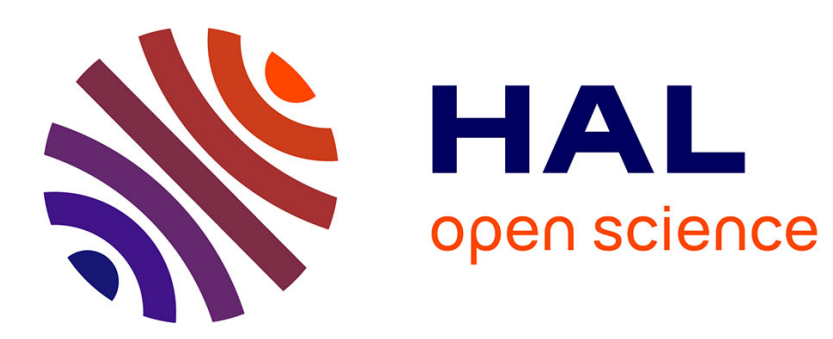

\title{
Propagation of two independent sources of uncertainty in the electrocardiography imaging inverse solution
}

\author{
Najib Fikal, Rajae Aboulaich, Emahdi El Guarmah, Nejib Zemzemi
}

\section{To cite this version:}

Najib Fikal, Rajae Aboulaich, Emahdi El Guarmah, Nejib Zemzemi. Propagation of two independent sources of uncertainty in the electrocardiography imaging inverse solution. Mathematical Modelling of Natural Phenomena, 2019, 14 (2), 10.1051/mmnp/2018065 . hal-01923847

\section{HAL Id: hal-01923847 \\ https://hal.inria.fr/hal-01923847}

Submitted on 15 Nov 2018

HAL is a multi-disciplinary open access archive for the deposit and dissemination of scientific research documents, whether they are published or not. The documents may come from teaching and research institutions in France or abroad, or from public or private research centers.
L'archive ouverte pluridisciplinaire HAL, est destinée au dépôt et à la diffusion de documents scientifiques de niveau recherche, publiés ou non, émanant des établissements d'enseignement et de recherche français ou étrangers, des laboratoires publics ou privés. 


\title{
PROPAGATION OF TWO INDEPENDENT SOURCES OF UNCERTAINTY IN THE ELECTROCARDIOGRAPHY IMAGING INVERSE SOLUTION
}

\author{
Najib. Fikal ${ }^{1}$, Rajae Aboulaich, Emahdi El Guarmah ${ }^{1,2}$ and Nejib \\ ZEMZEMI $^{3}$
}

\begin{abstract}
This work investigates the effects of the inputs parameters uncertainties (organs conductivities, boundary data) on the electrocardiography (ECG) imaging problem. These inputs are very important for the construction of the torso potential for the forward problem and for the non-invasive electrical potential on the heart surface in the case of the inverse problem. We propose a new stochastic formulation allowing to combine both sources of errors. We formulate the forward and the inverse stochastic problems by considering the inputs parameters as random fields and a stochastic optimal control formulation. In order to quantify multiple independent sources of uncertainties on the forward and inverse solutions, we attribute suitable probability density functions for each randomness source, and apply stochastic finite elements based on generalized polynomial chaos method. The efficiency of this approach to solve the forward and inverse ECG problem and the usability to quantify the effect of organs conductivity and epicardial boundary data uncertainties in the torso are demonstrated through a number of numerical simulations on a $2 \mathrm{D}$ computational mesh of a realistic torso geometry.
\end{abstract}

Mathematics Subject Classification. 65N20, 65N21, 65N22, 65N25, 65N30, 60H35.

The dates will be set by the publisher.

\section{INTRODUCTION}

The electrocardiography imaging (ECGI) is an imaging modality for the diagnosis of cardiac arrhythmia. It allows to recover the distribution of the electrical potential on the heart surface

\footnotetext{
Keywords and phrases. electrocardiography imaging forward and inverse problems; stochastic finite elements; uncertainty quantification; ill posed problem; boundary value problem

1 Mohammed V University of Rabat, Mohammadia school of Engineering LERMA and LIRIMA Laboratories. Av. Ibn Sina Agdal, Rabat Morocco. e-mail: najib.fikal@gmail.com \& aboulaich@gmail.com

2 Royal Air School, Informatics and Mathematics Department DFST, BEFRA, POB40002, Marrakech, Morocco. e-mail: guarmah@gmail.com

3 INRIA Bordeaux Sud-Ouest, Carmen project 200 rue de la vieille tour 33405 Talence Cedex, France, IHU Liryc, Electrophysiology and heart modeling institute. Avenue du Haut-Lévêque, 33604 Pessac, France. email: nejib.zemzemi@inria.fr
} 
from electrical measurements on the body surface and information about the torso geometry of the patient. The forward problem in electrocardiography allows to construct an electrical potential on the body of a patient given the values of the electrical sources on the heart domain [20]. This problem is mathematically well posed as it solves a Laplace equation with a Dirichlet boundary condition on the heart and a zero Neumann boundary condition on the body surface. While the inverse problem aims to construct the heart electrical potential given two boundary conditions on the body surface. The first condition is the non-flux boundary condition and the second one is a Dirichlet boundary condition taking into acount the electrical measurement on the body surface. This Cauchy problem is known to be ill-posed since Hadamard [21]. Therefore, a naive attempt to reconstruct the unknown heart potential leads to a wrong result or large deviations from the real solution. Besides the ill-posedness nature of this inverse problem, other sources of errors are generally omitted. First, conductivities of different organs in the torso cage are simplified using a homogeneous torso which produces an inaccurate description of the inhomogeneous torso volume conductor [28. This allows to use meshless methods like the method of fundamental solutions but it reduces the complexity of the model and could have an effect on the final result as shown in [33, 7, 27. Although one could find in the literature values of the organs conductivities, those could vary from a reference to another [14, 16, 18, Thus, considering this variability means to consider an uncertainty on their values in the model. Second, a very considerable progress has been achieved in developing the accuracy of medical devices and measurement system in the two last decades. But when recording the electrical potential on the body surface, measurement devices could generate errors, due to misplacement of electrodes or the precision of the device itself. In order to take into account these uncertainties and estimate their effects on the model solution, one could reformulate the deterministic model into a stochastic problem where constant parameters become random fields. Various sensitivity analysis methods have been developed to solve the stochastic problem. However, Monte Carlo (MC) [10, 31, which has been intensively used for different applications. However, MC approach is not efficient to be applied on complex systems for which the computational time for an accurate solving of the deterministic model is long. Another type of technique relies on differential analysis methods like the Neumann and Taylor series [23, 22, which are highly qualified to directly calculate the sensitivity coefficient. But, they are mathematically impractical for non linear systems. To overcome those limitations, we need to deal with the input data as a stochastic process and solving the resulting stochastic computational system of equations. One of the most appropriate methods that could be used for solving stochastic complex problems is the stochastic finite elements method (SFEM). In order to describe the stochastic distribution of the parameter of interest, this method assumes particular probability density functions (PDFs) [5, 6, 25].

In this work we propose a new stochastic formulation allowing to combine two sources of uncertainty, assuming that they have different kind of variability, and acting independently from each other. Each one will be described by random fields, with known prior statistics. The goal in this framework is expressing uncertainty through a probabilistic model in order to quantify a statistical index of the system response, like mean or variance. At first we consider the case of the forward ECG problem solved via SFEM. The problem is governed by a stochastic elliptic equation. The considered sources of uncertainty are the organs conductivities and the epicardial potential data.

We use a Galerkin stochastic Method [5, 15, 26] allowing to convert the stochastic system to a deterministic one. Following [34, 35] each source of randomness will be expressed by a finite number of random variables that are mutually independent. Using the polynomial chaos (PC) or generalized polynomial chaos (gPC), allows to represent the uncertain parameters as well as the response of the system by a set of coefficients in a suitable random polynomial basis. Then, 
an explicit functional relationship between the independent random variables of each source of uncertainty and the solution is achieved. Then, the stochastic governing equations are transformed to a set of deterministic equations which can be readily discretized via standard numerical techniques.

In the second part of this work we deal with the stochastic inverse ECG problem with torso boundary data, conductivity properties are described by random fields. Indeed, the deterministic inverse problem of electrocardiography can be formulated as an optimal control problem constrained by PDEs, i.e. minimization of an objective functional subject to a set of PDEs as constraints 22. For the stochastic formulation of an optimal control problem we can see [29, 30. The theoretical framework and tools for such stochastic control problems and their approximation are now well-known 4, 24. On the other hand, several methods for carrying out sensitivity analysis exist, but they are not widely applied to complex systems due to their computational cost and difficulty of implementation. In the work by Cao et.al [11, 12, a stochastic optimization problem is solved using the Monte-Carlo (MC) approach. It usually takes hundreds, if not thousands, of samples (solutions of the state equation) to obtain a satisfactory approximation of the cost function, which often makes it prohibitively expensive. The Bayesian inference approach is mostly used for the estimation of parameters in the presence of noisy measurements, using known information about the parameters to create a prior distribution [13, 32 .

In order to solve the considered stochastic inverse problem, we use the PC as in the first part. The derivation of the optimal system, is analogous to the deterministic case in which an energy functional has been used [1, 2]. The novelty of this work compared to the work in [2] is that the control variables that we look for are in the stochastic space which was not the case in 2]. This would allow us to find not only the mean value of the epicardial potential on the heart boundary but also its standard deviation in each point in the heart surface. In other words, we will be able to quantify the propagation of uncertainty from the torso boundary to the heart surface taking into account the uncertainties in the torso volume conductivity and the uncertainties in the electrical signals measurements on the torso. The goal is to quantify the effect of each source of uncertainty on the inverse solution and identify the one that affects more the electrical signal reconstruction.

\section{FUnCTION SPACES AND NOTATION}

We give, in the following, a short overview of the notations and the definition of the stochastic Sobolev space used throughout this paper. Let $D$ be the spatial domain. $\Omega$ is a sample space that belongs to a probability space $(\Omega, A, P)$, where $A$ denotes a $\sigma$-algebra of subsets of $\Omega$ and $P$ denotes a probability measure. Following the theory of Wiener [34, Karniadakis [35, we can approximate any general second-order random process $X(\omega), \omega \in \Omega$, in terms of a collection of finite number of random variables. We represent this random process by a vector $\boldsymbol{\xi}=\boldsymbol{\xi}(\omega)=$ $\left(\xi_{1}(\omega), \ldots, \xi_{N}(\omega)\right) \in \mathbb{R}^{N}$, where $N$ is the dimension of the approximated stochastic space. We assume that each random variable is independent, its image space is given by $\Theta_{i} \equiv \xi_{i}(\Omega) \subset \mathbb{R}$. Each random variable is characterised by a probability density function (PDF) $\rho_{i}: \Theta_{i} \longrightarrow \mathbb{R}^{+}$, for $i=1, \ldots, N$. Then, we define the joint PDF of the random vector $\boldsymbol{\xi}$

$$
\rho(\boldsymbol{\xi})=\prod_{i=1}^{N} \rho_{i}\left(\xi_{i}\right) \quad \forall \boldsymbol{\xi} \in \Theta,
$$

where the support of $\rho$ is $\Theta=\prod_{i=1}^{N} \Theta_{i}$. The probability measure on $\Theta$ is $\rho(\boldsymbol{\xi}) d \boldsymbol{\xi}$. As commented in [35], this allows us to conduct numerical formulations in the finite dimensional (N-dimensional) random space $\Theta$.

In this paper we treat a stochastic problem of electrocardiography, we suppose that the conductivity parameter and the epicardial boundary data act like two different and independent sources of uncertainties, which will be represented by two random process. For the conductivity parameter 
we define the probability space (respectively the vector of random variables, PDF, the PDF support) with $\left(\Omega_{0}, A_{0}, P_{0}\right)$, (respectively $\left.\boldsymbol{\xi}_{\mathbf{0}}, \rho_{0}, \Theta_{0}\right)$ and with $\left(\Omega_{1}, A_{1}, P_{1}\right),\left(\right.$ respectively $\left.\boldsymbol{\xi}_{\mathbf{1}}, \rho_{1}, \Theta_{1}\right)$ for the epicardial data.

Let us denote $\Theta=\Theta_{0} \times \Theta_{1}$ and $L^{2}(\Theta)=L^{2}\left(\Theta_{0}\right) \times L^{2}\left(\Theta_{1}\right)$ the space of random variables $X$ with finite second moments:

$$
\mathbb{E}\left[X^{2}\left(\boldsymbol{\xi}_{\mathbf{0}}, \boldsymbol{\xi}_{\mathbf{1}}\right)\right]=\int_{\Theta_{1}}\left(\int_{\Theta_{0}} X^{2}\left(\boldsymbol{\xi}_{\mathbf{0}}, \boldsymbol{\xi}_{\mathbf{1}}\right) \rho\left(\boldsymbol{\xi}_{\mathbf{0}}\right) d \boldsymbol{\xi}_{\mathbf{0}}\right) \rho\left(\boldsymbol{\xi}_{\mathbf{1}}\right) d \boldsymbol{\xi}_{\mathbf{1}}<+\infty,
$$

where $\mathbb{E}[$.$] denotes the mathematical expectation operator. This space is a Hilbert space with$ respect to the inner product:

$$
\langle X, Y\rangle_{L^{2}(\Theta)}=\mathbb{E}[X Y]=\int_{\Theta_{1}}\left(\int_{\Theta_{0}} X Y\left(\boldsymbol{\xi}_{\mathbf{0}}, \boldsymbol{\xi}_{\mathbf{1}}\right) \rho\left(\boldsymbol{\xi}_{\mathbf{0}}\right) d \boldsymbol{\xi}_{\mathbf{0}}\right) \rho\left(\boldsymbol{\xi}_{\mathbf{1}}\right) d \boldsymbol{\xi}_{\mathbf{1}}
$$

Additionally, we consider a spatial domain $D$ and we define the tensor product Hilbert space $L^{2}(D) \otimes L^{2}(\Theta)$ of second-order random fields as:

$$
L^{2}(D) \otimes L^{2}(\Theta)=\left\{u: D \otimes \Theta \longrightarrow \mathbb{R}, \int_{\Theta_{1}}\left(\int_{\Theta_{0}} \int_{D}\left|u\left(x, \boldsymbol{\xi}_{\mathbf{0}}, \boldsymbol{\xi}_{\mathbf{1}}\right)\right|^{2} d x \rho\left(\boldsymbol{\xi}_{\mathbf{0}}\right) d \boldsymbol{\xi}_{\mathbf{0}}\right) \rho\left(\boldsymbol{\xi}_{\mathbf{1}}\right) d \boldsymbol{\xi}_{\mathbf{1}}<\infty\right\} .
$$

Analogously, we introduce the Sobolev functional space $H^{1}(D)$ of square integrable functions in $D$ with square integrable derivatives. We denote by $H_{0}^{1}(D)$ the subspace of functions in $H^{1}(D)$ with zero trace on the boundary. The tensor product spaces $H^{1}(D) \otimes L^{2}(\Theta)$ and $H_{0}^{1}(D) \otimes L^{2}(\Theta)$ can be defined as:

$$
\begin{gathered}
H^{1}(D) \otimes L^{2}(\Theta)=\left\{u \in L^{2}(D) \otimes L^{2}(\Theta) \mid \int_{\Theta_{1}}\left(\int_{\Theta_{0}}\|u\|_{H^{1}(D)}^{2} \rho\left(\boldsymbol{\xi}_{\mathbf{0}}\right) d \boldsymbol{\xi}_{\mathbf{0}}\right) \rho\left(\boldsymbol{\xi}_{\mathbf{1}}\right) d \boldsymbol{\xi}_{\mathbf{1}}<\infty\right\}, \\
H_{0}^{1}(D) \otimes L^{2}(\Theta)=\left\{u \in H^{1}(D) \otimes L^{2}(\Theta) \mid u=0 \text { on } \partial D\right\} .
\end{gathered}
$$

\section{Solving THE STOCHASTIC FORWARD PROBLEM OF ELECTROCARDIOGRAPHY}

In this section we give a broad outline of the stochastic approach followed to solve the forward problem with input data uncertainties [19, 2. For the space domain we segment a 2D slice of an MRI image of a 56-year old man (see Figure 1). Under our assumption the conductivity uncertainties and epicardial boundary data uncertainties do not interact, and they are supposed to be independent from each other, consequently we represent the stochastic forward solution of the Laplace equation as a random field depending on both kinds of uncertainties. We suppose that the conductivity parameter $(\sigma)$ depends on the space $(x)$ and on the stochastic variable $\left(\xi_{0}\right)$, and the boundary epicardial data $(f)$ depends on space $(x)$ and on a stochastic variable $\left(\xi_{1}\right)$. Thus, the solution of the Laplace equation will depend on space and on both stochastic variables: we denote it by $u\left(x, \xi_{0}, \xi_{1}\right)$. The stochastic forward problem of electrocardiography can be written as follows

$$
\left\{\begin{array}{l}
\nabla \cdot\left(\sigma\left(x, \xi_{0}\right) \nabla u\left(x, \xi_{0}, \xi_{1}\right)\right)=0 \quad \text { in } D \times \Omega, \\
u\left(x, \xi_{0}, \xi_{1}\right)=f\left(x, \xi_{1}\right) \quad \text { on } \Gamma_{\mathrm{i}} \times \Omega \\
\sigma\left(x, \xi_{0}\right) \frac{\partial u\left(x, \xi_{0}, \xi_{1}\right)}{\partial n}=0 \quad \text { on } \Gamma_{\mathrm{c}} \times \Omega
\end{array}\right.
$$

where $\Gamma_{\mathrm{i}}$ and $\Gamma_{\mathrm{c}}$ represent the epicardial and torso boundaries respectively. The weak formulation of SPDEs is based on an extension of the deterministic theory [5], test functions become random fields and an integration over stochastic space is done with respect to the corresponding measure. 

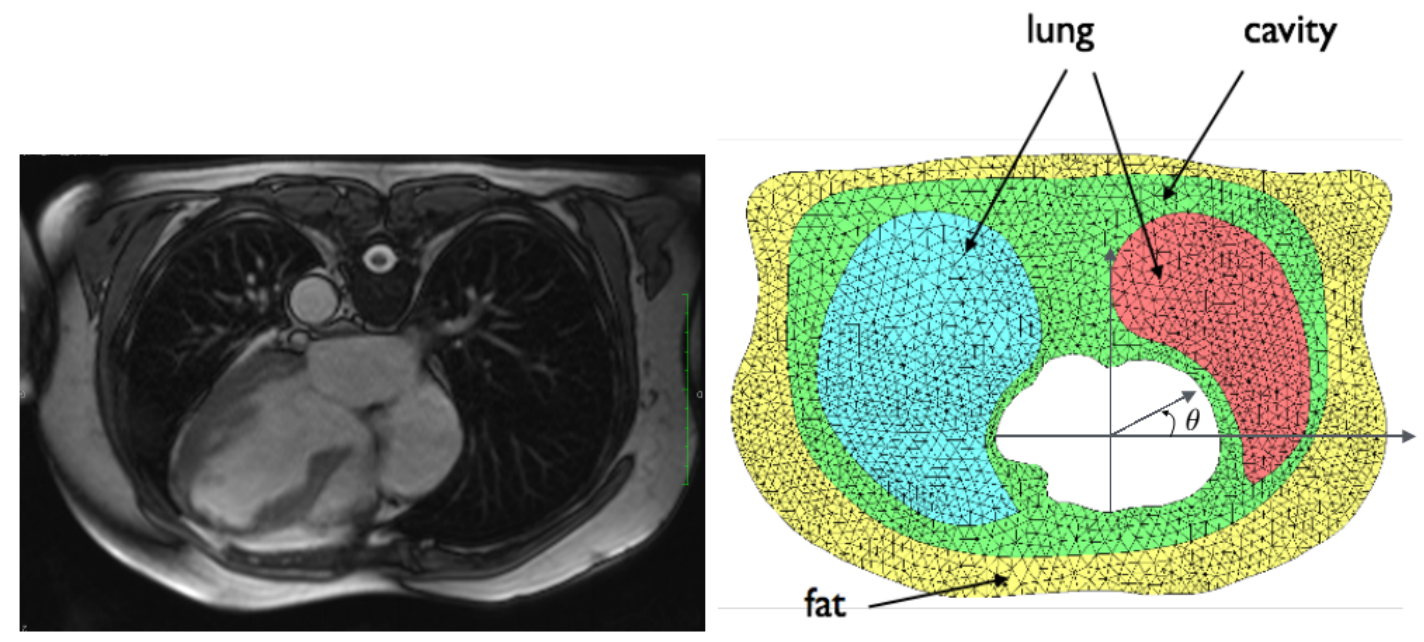

FIGURE 1. MRI 2D slice of the torso (left), 2D computational mesh of the torso geometry showing the different regions of the torso considered in this study: fat, lungs and torso cavity (right). The angle $\theta$ is the second polar coordinate.

Thus, the weak form involves expectations of the weak problem formulation in the physical space. Then, denoting by $u_{f}$ the extension of $f$ to the whole domain, we look for $\tilde{u} \in H_{0 f}^{1}(D) \otimes L^{2}(\Theta)$ with:

$$
H_{0 f}^{1}(D) \otimes L^{2}(\Theta)=\left\{u \in H^{1}(D) \otimes L^{2}(\Theta) \mid u=0 \text { on } \Gamma_{c}\right\} .
$$

Where $\tilde{u}=u-u_{f}$ is the weak solution of (1), if for all $v \in H_{0 f}^{1}(D) \otimes L^{2}(\Theta)$, we have:

$\mathbb{E}\left[\int_{D} \sigma\left(x, \xi_{1}\right) \nabla \tilde{u}\left(x, \xi_{0}, \xi_{1}\right) \cdot \nabla v\left(x, \xi_{0}, \xi_{1}\right) d x\right]+\mathbb{E}\left[\int_{D} \sigma\left(x, \xi_{1}\right) \nabla u_{f}\left(x, \xi_{0}\right) \cdot \nabla v\left(x, \xi_{0}, \xi_{1}\right) d x\right]=0$

\subsection{Discretization of the stochastic forward problem}

A square integrable stochastic process $X(\xi)$ of a parameter or a variable $X$ is represented by weighted sum of orthogonal polynomials $\left\{\Psi_{i}(\xi)\right\}$ denoting the generalized chaos polynomial. More details about the different choices of PDFs could be found in [25].

$$
X(\xi)=\hat{X}_{0}+\sum_{i=1}^{\infty} \hat{X}_{i} \Psi_{i}(\xi)
$$

where $\hat{X}_{i}$ are the projections of the random process on the stochastic basis $\left\{\Psi_{i}(\xi)\right\}_{i=0}^{\infty}$ with respect to the joint $\mathrm{PDF} \rho[19]$. In practice, the infinite sum is truncated at a given order $p$ that allows a sufficient accuracy. We denote by

$$
\hat{X}(\xi)=\hat{X}_{0}+\sum_{i=1}^{p} \hat{X}_{i} \Psi_{i}(\xi)
$$

The mean value and the standard deviation of $\hat{X}$ over $\Omega$ are then computed as follows: 


$$
\mathbb{E}(\hat{X})=\int_{\Omega} \sum_{i=0}^{p} \hat{X}_{i} \Psi_{i}(\xi)=\hat{X}_{0}, \operatorname{stdev}[\hat{X}]=\left(\sum_{i=1}^{p} \hat{X}_{i}^{2} \int_{\Omega} \Psi_{i}(\xi)^{2}\right)^{\frac{1}{2}} .
$$

In order to solve the equation (1) we use the stochastic Galerkin (SG) method to compute the approximate solutions. To develop this method, we denote $Y_{\sigma}^{p} \subset L^{2}\left(\Theta_{0}\right)$ and $Y_{u_{f}}^{p} \subset L^{2}\left(\Theta_{1}\right)$ the stochastic approximation spaces, and we have $Y_{\sigma}^{p} \times Y_{u_{f}}^{q} \subset L^{2}(\Theta)$.

In our case we suppose that the conductivity parameter varies uniformly like in [19, 2] and we use the Legendre chaos polynomials which are more suitable for uniform probability density. On the other hand, we assigned Gaussian probability density to the epicardial boundary data. The corresponding stochastic orthogonal basis to Gaussian random field is Hermite chaos polynomials [35].

$$
Y_{\sigma}^{P}=\operatorname{span}\left\{L_{0}, \ldots, L_{p}\right\} ; Y_{u_{f}}^{P}=\operatorname{span}\left\{H_{0}, \ldots, H_{p}\right\} .
$$

In this study, our aim is to evaluate, at the same time, two different sources of uncertainties on the electrical potential, We denote by $\hat{\sigma}, \hat{u}_{f}$ and $\hat{u}$ the Galerkin approximation of $\sigma, u_{f}$ and $u$, respectively in the spaces $Y_{\sigma}^{p}, Y_{u_{f}}^{q}$ and $Y_{\sigma}^{p} \times Y_{u_{f}}^{q}$ respectively. They are given by

$$
\begin{gathered}
\sigma\left(x, \xi_{0}\right)=\sum_{l=1}^{p} \hat{\sigma}_{l}(x) L_{l}\left(\xi_{0}\right) . \\
u_{f}\left(x, \xi_{1}\right)=\sum_{k=1}^{q}\left(\tilde{u}_{f}\right)_{k}(x) H_{k}\left(\xi_{1}\right) . \\
u\left(x, \xi_{0}, \xi_{1}\right)=\sum_{i=1}^{p} \sum_{j=1}^{q} \hat{u}_{i j}(x) L_{i}\left(\xi_{0}\right) H_{j}\left(\xi_{1}\right)
\end{gathered}
$$

By substituting (6), (5), (7) into the stochastic diffusion equation (1) and by projecting the result on the polynomial basis $\left\{L_{m}\left(\xi_{0}\right) H_{n}\left(\xi_{1}\right)\right\}_{m, n=1}^{(p, q)}$ :

For $m=1, \ldots, q$ and $n=1, \ldots, p$,

$$
\begin{array}{rr}
\sum_{i=1}^{p} \sum_{j=1}^{q} \sum_{l=1}^{p} & \left.D_{j n} C_{i m l} \nabla \cdot\left(\hat{\sigma}_{l}(x) \nabla\right) \hat{u}_{i j}(x)\right)=0 \text { in } D, \\
\forall k=1, \ldots, q, & \forall i=2, \ldots, p \text { and } j=1, \ldots, q, \\
\hat{u}_{1 k}(x)=\left(\tilde{u}_{f}\right)_{k}(x) \text { on } \Gamma_{\mathrm{i}}, & \forall i=1, \ldots, p \text { and } j=1, \ldots q, \\
\hat{u}_{i j}(x)=0 \text { on } \Gamma_{\mathrm{i}}, &
\end{array}
$$

where $C_{i m l}=\mathbb{E}\left[L_{i}\left(\xi_{0}\right), L_{m}\left(\xi_{0}\right), L_{l}\left(\xi_{0}\right)\right]$ et $D_{j n}=\mathbb{E}\left[H_{j}\left(\xi_{1}\right), H_{n}\left(\xi_{1}\right)\right]$.

For the spatial domain, we define a subspace $V_{h} \subset H_{0 f}^{1}(D)$ of standard Lagrange finite element functions on a triangulation of the domain $D$.

$$
V_{h}:=\operatorname{span}\left\{\phi_{1}, \phi_{2}, \ldots, \phi_{N}\right\}
$$


Obviously this ordering induces the following block structure of the linear system of equations :

$$
\left[\begin{array}{ccccccc}
A^{(1,1 ; 1,1)} & A^{(1,1 ; 1,2)} & \cdots & A^{(1,1 ; 1, q)} & A^{(1,1 ; 2,1)} & \cdots & A^{(1,1 ; p, q)} \\
A^{(1,2 ; 1,1)} & A^{(1,2 ; 1,2)} & \cdots & A^{(1,2 ; 1, q)} & A^{(1,2 ; 2,1)} & \cdots & A^{(1,2 ; p, q)} \\
\vdots & \vdots & \cdots & \vdots & \vdots & \cdots & \vdots \\
A^{(1, q ; 1,1)} & A^{(1, q ; 1,2)} & \cdots & A^{(1, q ; 1, q)} & A^{(1, q ; 2,1)} & \cdots & A^{(1, q ; p, q)} \\
A^{(2,1 ; 1,1)} & A^{(2,1 ; 1,2)} & \cdots & A^{(2,1 ; 1, q)} & A^{(2,1 ; 2,1)} & \cdots & A^{(2,1 ; p, q)} \\
\vdots & \vdots & \cdots & \vdots & \vdots & \cdots & \vdots \\
A^{(p, q ; 1,1)} & A^{(p, q ; 1,2)} & \cdots & A^{(p, q ; 1, q)} & A^{(p, q ; 2,1)} & \cdots & A^{(p, q ; p, q)}
\end{array}\right]\left[\begin{array}{c}
\hat{U}_{11} \\
\hat{U}_{12} \\
\vdots \\
\hat{U}_{1 q} \\
\hat{U}_{21} \\
\vdots \\
\hat{U}_{p q}
\end{array}\right]=\left[\begin{array}{c}
B^{11} \\
B^{12} \\
\vdots \\
B^{1 q} \\
B^{21} \\
\vdots \\
B^{p q}
\end{array}\right]
$$

where every matrix $A^{(i, j ; m, n)} \in \mathbb{R}^{N x} \times \mathbb{R}^{N x}$ is a linear combination of finite element stiffness matrices

$$
\begin{gathered}
A^{(i, j ; m, n)}=D_{j, n} \sum_{l=1}^{p} C_{i m l} K_{l} \quad \forall i, m=1, \ldots p ; j, n=1, \ldots q, \\
K_{l}=\left[K_{l}\right]_{h, t}=\left(\hat{\sigma}_{l} \nabla \phi_{h} . \nabla \phi_{t}\right)_{1 \leq h, t \leq N} \quad \forall l=1, \ldots p,
\end{gathered}
$$

Here, $h$ and $t$ denote the degrees of freedom of the nodes of the mesh in which the electrical potential values is unknown.

Similarly, every vector $B^{i j} \in \mathbb{R}^{N}$ is a linear combination of finite element load vectors:

$$
B^{i j}=\sum_{n}^{q} \sum_{m}^{p} \sum_{l=1}^{p} D_{j, n} C_{i m l} f_{l} \quad \forall i=1, \ldots p, j=1, \ldots q,
$$

where the vector $f_{l} \in \mathbb{R}^{N}$ is given by

$$
f_{l}=\sum_{x_{h} \in \Gamma_{\mathrm{i}}}\left(\tilde{u}_{f}\right)_{l}\left(x_{h}\right)\left(\hat{\sigma}_{l} \nabla \phi_{h} . \nabla \phi_{t}\right)_{1 \leq t \leq N} \quad \forall l=1, \ldots p .
$$

The index $h$ denotes the degrees of freedom of the known Dirichlet boundary conditions of the solution.

\subsection{Results}

In this section we conduct the numerical simulation obtained for the direct problem, which show the influence of the conductivity variabilities and the epicardial potential data uncertainties on the electrical potential in the torso. For the electrical potential in the heart boundary denoted by $\left(U_{e x}\right)$, we generate synthetical data using the bidomain model in the heart domain [17.

Since we assume that the uncertainty of the conductivity value follows a uniform probability density, as probability density functions $\rho_{0}$ we use the Legendre polynomials defined on the interval $\Omega=[-1,1]$. We also suppose that the true conductivity uncertainty interval is centered by $\sigma_{T}$, the true conductivity see Table 1 . On the other hand $U_{e x}$ will represent the mean of the Gaussian random field representing the epicardial boundary data uncertainty. We denote its stdev by $\kappa$ with $(\kappa=\nu f), \nu$ represents the level of uncertainty of the torso data. Under these assumptions, we express the conductivity and the epicardial boundary data as follows [8, 19]:

$$
\begin{aligned}
\hat{\sigma}\left(x, \xi_{0}\right) & =\hat{\sigma}_{0}(x) L_{0}\left(\xi_{0}\right)+\hat{\sigma}_{1}(x) L_{1}\left(\xi_{0}\right) . \\
\hat{u}_{f}\left(x, \xi_{1}\right) & =\tilde{u}_{f_{0}}(x) H_{0}\left(\xi_{1}\right)+\tilde{u}_{f_{1}}(x) H_{1}\left(\xi_{1}\right) .
\end{aligned}
$$




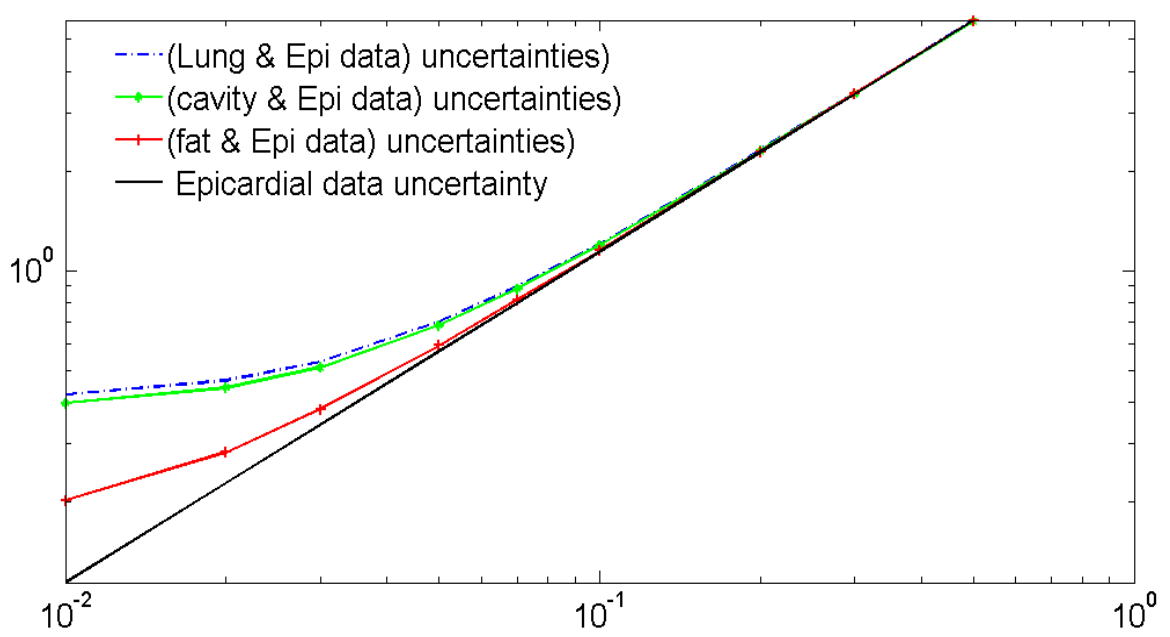

FIgURE 2. The effects of $\pm 50 \%$ uncertainty to each organ conductivity from its reference conductivity, and different levels of uncertainty on the epicardial boundary data. $\mathrm{X}$-axis denote the different stdev value $(\nu)$ of the Gaussian epicardial data boundary field. Y-axis the mean square of the different stdev value of $u\left(x, \xi_{0}, \xi_{1}\right)$.

where $\sigma\left(x, \xi_{0}\right)$ is uniformly distributed on the interval $[a(x), b(x)]$ for each point $x \in D$. We have then $\hat{\sigma}_{0}(x)=0,5(a(x)+b(x))$ and $\hat{\sigma}_{1}(x)=0,5(b(x)-a(x))$. For $u_{f}\left(x, \xi_{1}\right)$ which follows a Gaussian distribution for each point $x \in \Gamma_{c}$, we have $\tilde{u}_{f_{0}}=U_{e x}$ and $\tilde{u}_{f_{1}}=\kappa$.

Figure 2 shows the set of results obtained for the relative error between the exact solution

\begin{tabular}{|c|c|}
\hline organ category & conductivity $\left(\sigma_{T}: \mathrm{S} / \mathrm{m}\right)$ \\
\hline lungs & 0.096 \\
torso cavity & 0.200 \\
fat & 0.045 \\
\hline
\end{tabular}

TABLE 1. Conductivity values corresponding to the organs that are considered in the model.

and the obtained solution on $\Gamma_{\mathrm{c}}$. In the first case we only study the effect of torso boundary data uncertainties where we gradually increase the stdev $\nu$ from zero to $50 \%$. In the second (respectively, third, fourth) case we add the effect of fat (respectively, cavity, lung) with $\pm 50 \%$ of uncertainties. We find that the relative error(RE) of the forward problem solution has been barely affected by the uncertainties of the fat conductivity giving a relative error equal to $2 \%$. On the contrary the effect of the lung and cavity conductivity uncertainties is high( $\mathrm{RE}=12 \%)$, and stay observable to some level of $\nu \geq 10^{-1}$, in which all curves take the same values as the case with only epicardial boundary data uncertainty. Figure 3 approves the obtained results and analysis mentioned above in Figure 2, and illustrates the uncertainty propagation in the whole domain. As seen the lung and the torso boundary data uncertainties case Figure $3(\mathrm{e})$ take the same distribution as the case with only epicardial boundary data uncertainty Figure 3 (f). 


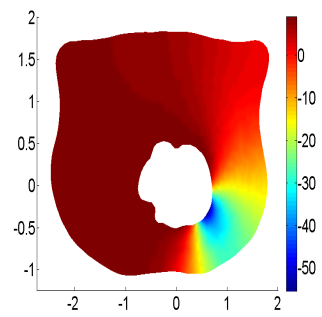

(a)

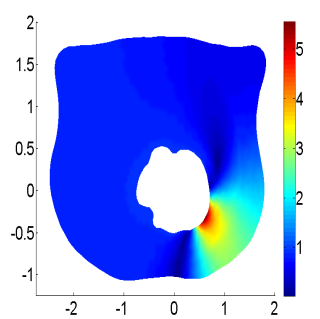

(d)

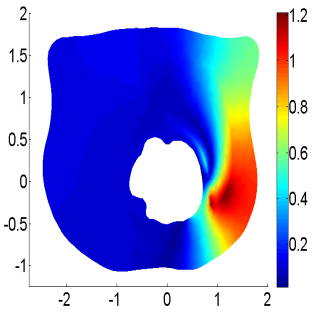

(b)

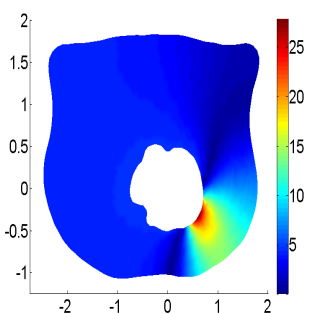

(e)

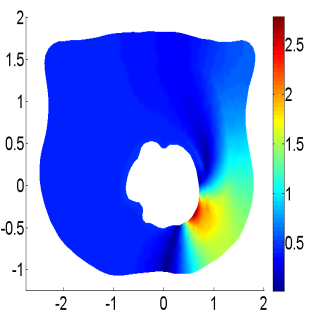

(c)

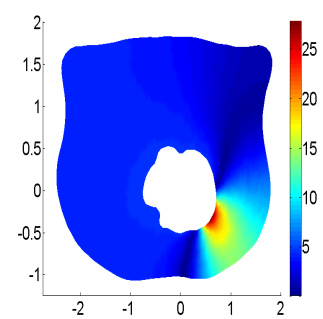

(f)

Figure 3. Mean value of the SFE panel (a). Standard deviation of the SFE solution for epicardial data uncertainty in the left and in the right $\pm 50 \%$ lung uncertainty and epicardial data uncertainty for $\nu=0.01$ (respectively from top the bottom $\nu=0.1, \nu=0.3 \operatorname{panel}(\mathrm{d}), \nu=0.5 \operatorname{panel}(\mathrm{e}))$. Panel(f) shows the Standard deviation of the SFE solution with only epicardial data uncertainty for $\nu=0.5$

\section{STOCHASTIC INVERSE PROBLEM OF ELECTROCARDIOGRAPHY}

The inverse problem in electrocadiography imaging (ECGI) is a technique that allows to construct the electrical potential on the heart surface $\Gamma_{\mathrm{i}}$ from data measured on the body surface $\Gamma_{\mathrm{c}}$. This problem is known to be ill-posed since Hadamard [21, then we propose to build an optimal control problem, we write a cost function that takes into account the uncertainties in the torso conductivities and the torso boundary data. We then use an energy cost function as used in [1, constrained by Laplace formulation as presented in the previous paragraph, with conductivity and torso boundary data being stochastic processes. We look for $(\eta, \tau) \in\left(L^{2}\left(\Gamma_{\mathrm{i}}\right) \otimes\right.$ $\left.L^{2}(\Theta)\right) \times\left(L^{2}\left(\Gamma_{\mathrm{i}}\right) \otimes L^{2}(\Theta)\right)$ minimizing the following cost function with a least square setting:

$$
\left\{\begin{aligned}
J(\eta, \tau)=\frac{1}{2} \mathbb{E}\left(\int_{\Gamma_{\mathrm{c}}}\left(v\left(x, \xi_{0}, \xi_{1}\right)-f\right)^{2} d x\right. & \\
& \left.\quad+\int_{\Gamma_{\mathrm{i}}}\left(\sigma\left(x, \xi_{0}\right) \frac{\partial v\left(x, \xi_{0}, \xi_{1}\right)}{\partial n}-\eta\right)^{2} d x\right), \\
& \text { with } v\left(x, \xi_{0}, \xi_{1}\right) \text { solution of : } \\
\nabla \cdot\left(\sigma\left(x, \xi_{0}\right) \nabla v\left(x, \xi_{0}, \xi_{1}\right)\right)=0, & \text { in } D \times \Omega, \\
v\left(x, \xi_{0}, \xi_{1}\right)=\tau, & \text { on } \Gamma_{\mathrm{i}} \times \Omega, \\
\sigma\left(x, \xi_{0}\right) \frac{\partial v\left(x, \xi_{0}, \xi_{1}\right)}{\partial n}=0, & \text { on } \Gamma_{\mathrm{c}} \times \Omega .
\end{aligned}\right.
$$


We followed the same way presented in our last work [2], to calculate the gradient of the cost function $J$ with respect to $\eta=\eta_{0}(x) H_{0}\left(\xi_{1}\right)+\eta_{1}(x) H_{1}\left(\xi_{1}\right)$, and $\tau=\tau_{0}(x) H_{0}\left(\xi_{1}\right)+\tau_{1}(x) H_{1}\left(\xi_{1}\right)$, the obtained result is given by:

$$
\begin{cases}\frac{\partial J(\eta, \tau)}{\partial \eta_{0}} \phi_{0}=-\mathbb{E}\left[\int_{\Gamma_{\mathrm{i}}}\left(\sigma \frac{\partial v}{\partial n}-\eta\right) \phi_{0} d x\right], & \forall \phi_{0} \in L^{2}\left(\Gamma_{\mathrm{i}}\right), \\ \frac{\partial J(\eta, \tau)}{\partial \tau_{0}} h_{0}=\mathbb{E}\left[\int_{\Gamma_{\mathrm{i}}} \sigma \frac{\partial \lambda}{\partial n} h_{0} d x\right], & \forall h_{0} \in L^{2}\left(\Gamma_{\mathrm{i}}\right), \\ \frac{\partial J(\eta, \tau)}{\partial \eta_{1}} \phi_{1}=-\mathbb{E}\left[\int_{\Gamma_{\mathrm{i}}}\left(\sigma \frac{\partial v}{\partial n}-\eta\right) \phi_{1} d x\right], & \forall \phi_{1} \in L^{2}\left(\Gamma_{\mathrm{i}}\right), \\ \frac{\partial J(\eta, \tau)}{\partial \tau_{1}} h_{1}=\mathbb{E}\left[\int_{\Gamma_{\mathrm{i}}} \sigma \frac{\partial \lambda}{\partial n} h_{1} d x\right], & \forall h_{1} \in L^{2}\left(\Gamma_{\mathrm{i}}\right), \\ \text { with } \lambda \text { solution of : } & \\ \nabla \cdot\left(\sigma\left(x, \xi_{0}\right) \nabla \lambda\left(x, \xi_{0}, \xi_{1}\right)\right)=0, & \text { on } D \times \Omega, \\ \lambda\left(x, \xi_{0}, \xi_{1}\right)=\sigma\left(x, \xi_{0}\right) \frac{\partial v\left(x, \xi_{0}, \xi_{1}\right)}{\partial n}-\eta, & \text { on } \Gamma_{\mathrm{i}} \times \Omega, \\ \sigma\left(x, \xi_{0}\right) \frac{\partial \lambda\left(x, \xi_{0}, \xi_{1}\right)}{\partial n}=-\left(v\left(x, \xi_{0}, \xi_{1}\right)-f\left(x, \xi_{1}\right)\right), & \text { on } \Gamma_{\mathrm{c}} \times \Omega .\end{cases}
$$

\subsection{The conjugate gradient algorithm.}

Let us solve numerically the minimization problem 15 . As method, we choose the conjugate gradient optimization procedure, which is one of the most intuitive and simple methods used to solve problems of optimal control [36, 1]. The different steps of the algorithm are performed as follows:

Step 1. Given $f \in L^{2}\left(\Gamma_{\mathrm{c}}\right) \otimes L^{2}(\Theta)$ choose an arbitrary initial guess

$$
\left(\varphi_{p}, t_{p}\right) \in\left(L^{2}\left(\Gamma_{\mathrm{i}}\right) \otimes L^{2}(\Theta)\right) \times\left(L^{2}\left(\Gamma_{\mathrm{i}}\right) \otimes L^{2}(\Theta)\right) .
$$

Step 1.1. Solve the well-posed stochastic forward problem:

$$
\begin{cases}\nabla \cdot\left(\sigma\left(x, \xi_{0}\right) \nabla v^{p}\left(x, \xi_{0}, \xi_{1}\right)\right)=0 & \text { in } D \times \Omega, \\ \sigma\left(x, \xi_{0}\right) \frac{\partial v^{p}\left(x, \xi_{0}, \xi_{1}\right)}{\partial n}=0 & \text { on } \Gamma_{\mathrm{c}} \times \Omega \\ v^{p}\left(x, \xi_{0}, \xi_{1}\right)=t_{p} & \text { on } \Gamma_{\mathrm{i}} \times \Omega\end{cases}
$$

in order to obtain $v^{p} \mid \Gamma_{\mathrm{i}}$ and $\sigma \frac{\partial v^{p}}{\partial n} \mid \Gamma_{\mathrm{i}}$.

Step 1.2. Solve the stochastic adjoint problem:

$$
\begin{cases}\nabla \cdot\left(\sigma\left(x, \xi_{0}\right) \nabla \lambda^{p}\left(x, \xi_{0}, \xi_{1}\right)\right)=0 & \text { in } \quad D \times \Omega \\ \lambda^{p}\left(x, \xi_{0}, \xi_{1}\right)=\sigma\left(x, \xi_{0}\right) \frac{\partial v^{p}(x, \xi)}{\partial n}-\varphi_{p} & \text { on } \quad \Gamma_{\mathrm{i}} \times \Omega \\ \sigma\left(x, \xi_{0}\right) \frac{\partial \lambda^{p}\left(x, \xi_{0}, \xi_{1}\right)}{\partial n}=-\left(v^{p}\left(x, \xi_{0}, \xi_{1}\right)-f\left(x, \xi_{1}\right)\right) & \text { on } \quad \Gamma_{\mathrm{c}} \times \Omega\end{cases}
$$

in order to obtain $\lambda^{p} \mid \Gamma_{\mathrm{i}}$, and $\sigma \frac{\partial \lambda^{p}}{\partial n} \mid \Gamma_{\mathrm{i}}$.

Step 1.3.: We evaluate the gradient:

$$
\begin{aligned}
& \nabla J\left(\varphi_{p}^{0}, t_{p}^{0}\right)=\left(\mathbb{E}\left[\varphi_{p}^{0}-\sigma\left(x, \xi_{0}\right) \frac{\partial v^{p}\left(x, \xi_{0}, \xi_{1}\right)}{\partial n}\right], \mathbb{E}\left[\sigma\left(x, \xi_{0}\right) \frac{\partial \lambda_{00}^{p}\left(x, \xi_{0}, \xi_{1}\right)}{\partial n}\right]\right), \\
& \nabla J\left(\varphi_{p}^{1}, t_{p}^{1}\right)=\left(\mathbb{E}\left[\varphi_{p}^{1}-\sigma\left(x, \xi_{0}\right) \frac{\partial v^{p}\left(x, \xi_{0}, \xi_{1}\right)}{\partial n}\right], \mathbb{E}\left[\sigma\left(x, \xi_{0}\right) \frac{\partial \lambda_{01}^{p}\left(x, \xi_{0}, \xi_{1}\right)}{\partial n}\right]\right) .
\end{aligned}
$$


Step 1.4. Determine the descent direction $d_{p}$ as follows:

in order to obtain:

$$
\left\{\begin{array}{l}
\gamma_{p-1}^{0}=\frac{\left\|\nabla J\left(\varphi_{0}^{p}, t_{0}^{p}\right)\right\|^{2}}{\left\|\nabla J\left(\varphi_{0}^{p-1}, t_{0}^{p-1}\right)\right\|^{2}}, \\
\gamma_{p-1}^{1}=\frac{\left\|\nabla J\left(\varphi_{1}^{p}, t_{1}^{p}\right)\right\|^{2}}{\left\|\nabla J\left(\varphi_{1}^{p-1}, t_{1}^{p-1}\right)\right\|^{2}}, \\
d_{p}^{0}:=\left(d_{10}^{p}, d_{20}^{p}\right)=-\nabla J\left(\varphi_{0}^{p}, t_{0}^{p}\right)+\gamma_{p-1}^{0} d_{p-1}^{0}, \\
d_{p}^{1}:=\left(d_{11}^{p}, d_{20}^{p}\right)=-\nabla J\left(\varphi_{1}^{p}, t_{1}^{p}\right)+\gamma_{p-1}^{1} d_{p-1}^{1},
\end{array}\right.
$$

$$
\begin{aligned}
& \left(\varphi_{p+1}^{0}, t_{p+1}^{0}\right)=\left(\varphi_{p}^{0}, t_{p}^{0}\right)+\alpha_{p}^{0} d_{0}^{p} \\
& \left(\varphi_{p+1}^{1}, t_{p+1}^{1}\right)=\left(\varphi_{p}^{1}, t_{p}^{1}\right)+\alpha_{p}^{1} d_{1}^{p}
\end{aligned}
$$

where the scalar $\alpha_{p}$ is obtained through a linear search by :

$$
\begin{aligned}
\alpha_{p}^{0} & =-\frac{\mathbb{E}\left[\int_{\Gamma_{\mathrm{c}}} z^{p}\left(v^{p}-f\right) d x\right]+\mathbb{E}\left[\int_{\Gamma_{\mathrm{i}}}\left(\sigma \frac{\partial z^{p}}{\partial n}-d_{10}^{p}\right)\left(\sigma \frac{\partial v^{p}}{\partial n}-\varphi_{p}^{0}\right) d x\right]}{\mathbb{E}\left[\int_{\Gamma_{\mathrm{c}}}\left(z^{p}\right)^{2} d x\right]+\mathbb{E}\left[\int_{\Gamma_{\mathrm{i}}}\left(\sigma \frac{\partial z^{p}}{\partial n}-d_{10}^{p}\right)^{2} d x\right]}, \\
\alpha_{p}^{1} & =-\frac{\mathbb{E}\left[\int_{\Gamma_{\mathrm{c}}} z^{p}\left(v^{p}-f\right) d x\right]+\mathbb{E}\left[\int_{\Gamma_{\mathrm{i}}}\left(\sigma \frac{\partial z^{p}}{\partial n}-d_{11}^{p}\right)\left(\sigma \frac{\partial v^{p}}{\partial n}-\varphi_{p}^{1}\right) d x\right]}{\mathbb{E}\left[\int_{\Gamma_{\mathrm{c}}}\left(z^{p}\right)^{2} d x\right]+\mathbb{E}\left[\int_{\Gamma_{\mathrm{i}}}\left(\sigma \frac{\partial z^{p}}{\partial n}-d_{11}^{p}\right)^{2} d x\right]} .
\end{aligned}
$$

We note that $z_{p}$ is the solution of:

$$
\begin{cases}\nabla \cdot\left(\sigma\left(x, \xi_{0}\right) \nabla z^{p}\left(x, \xi_{0}, \xi_{1}\right)\right)=0 & \text { in } D \times \Omega, \\ \sigma\left(x, \xi_{0}\right) \frac{\partial z^{p}\left(x, \xi_{0}, \xi_{1}\right)}{\partial n}=0 & \text { on } \Gamma_{\mathrm{c}} \times \Omega \\ z^{p}\left(x, \xi_{0}, \xi_{1}\right)=d_{1}^{p} & \text { on } \Gamma_{\mathrm{i}} \times \Omega .\end{cases}
$$

Step 2. Having obtained $\left(\varphi_{p}, t_{p}\right)$ for $p \geq 0$, set $p=p+1$ and repeat from step 1.1 until the prescribed stopping criterion is satisfied. As a stopping criterion, we choose $\left.\| \mathbb{E}\left[t^{p+1}(x, \xi)\right]-t^{p}(x, \xi)\right] \|_{L^{2}\left(\Gamma_{\mathrm{i}}\right)} \leq$ $\epsilon$. In practice, we took $\epsilon=0.001$.

\subsection{Results}

In this paragraph, we show the numerical results of the stochastic inverse problem. Initially, we generate synthetical data using the bidomain model in the heart domain. Since we suppose that the torso is a passive conductor, the electrical potential in the torso is governed by the Laplace equation and the conductivity depends on the domain as described. We extract the body surface potential at a given time step, it represents the mean of the stochastic boundary value $\mathbb{E}(f)$ on the complete boundary $\Gamma_{c}$. Then, we solve the inverse problem following the algorithm described in previous paragraph.

In order to display the stochastic behavior of the reconstructed epicardial potential $\tau$, we run various simulations for different levels of $\nu$. Figure 4 provides an overview of the obtained results taking into account solely the torso data as source of uncertainty. Figure 4(a) gives the obtained result for $\nu=1 \%$ for $(3,100,500$ and 1000) samples of $\tau$, in Figure 4(b) (respectively, Figure 4(c), Figure 4(d)), we do the same for $\nu=10 \%$ (respectively,30\%, 50\%). Analyzing these results, it is observed that reconstructed epicardial potential $\tau$ at a level of $\nu$ less than $10 \%$ still have a homogenous deviation to the exact solution with greater margin of error compared to the first case $\nu=1 \%$ in which the effect appears negligible. Though once $\nu$ exceeds $10 \%$, the effect of the 
torso uncertainty seems more and more important, and produces a large variability on $\tau$.

Therefore, to bring out the effect of the organs conductivity uncertainties, Figure 5 summarizes the obtained results combining the torso data and the organs conductivity uncertainties. From top to bottom $\nu=1 \%, 10 \%, 30 \%$ and $50 \%$, we show the mean value of the SFEM inverse solution $\mu$ (black line), exact solution (blue dashed line) and the $95 \%$ confidence interval (cyan region bounded by red lines). For each point on the heart boundary the $95 \%$ confidence interval is defined as $[\mu-2 \mathrm{stdev}, \mu+2 \mathrm{stdev}]$. In this figure, there are four columns: In the first column, we only consider the uncertainty on the measured data $f$ and in the second (respectively, third, fourth) we consider both uncertainties on the measured data $f$ and $50 \%$ uncertainty on the fat (respectively, cavity, lung) conductivity. First, we remark that increasing the level of $\nu$ from $1 \%$ to $30 \%$, the epicardial data $(\tau)$ is more sensitive to the cavity and lung conductivities than for fat, as found in the numerical results obtained in [2]. Then, we observe that the organs conductivity effects become slightly important, once the value of $\nu$ exceeds $30 \%$. As shown in the two cases with $\nu=30 \%$ (third line) and $\nu=50 \%$ (fourth line), the error of the inverse mean value compared to the exact solution is more or less the same for the four uncertainty cases considered here. 

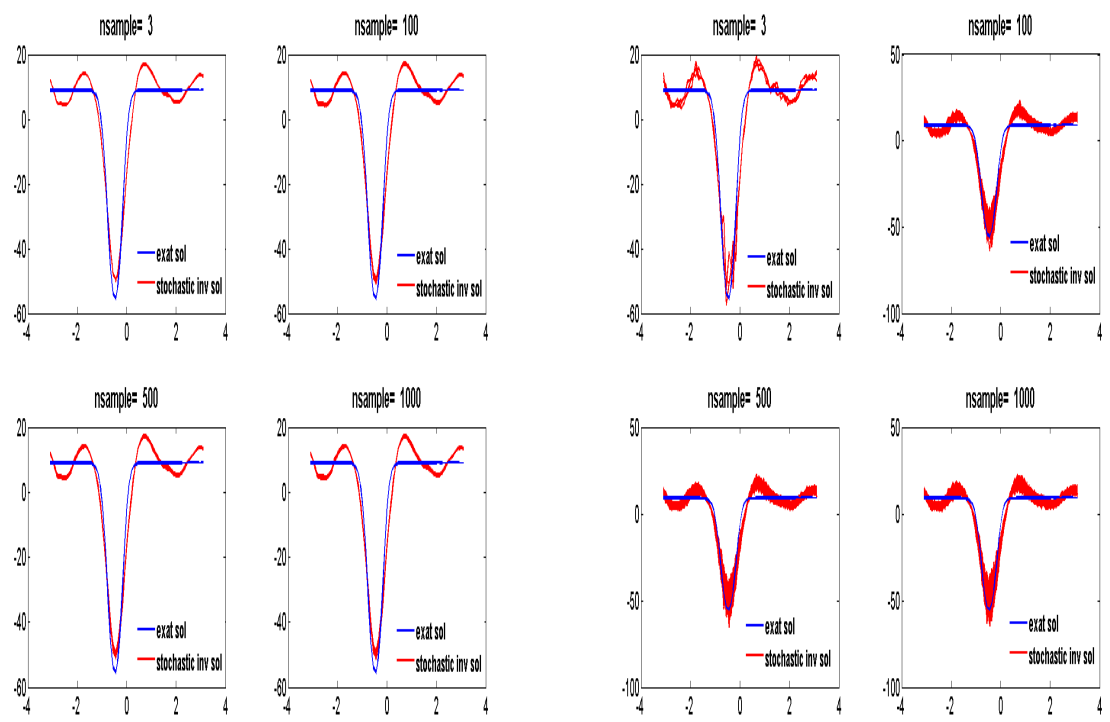

(a)

(b)
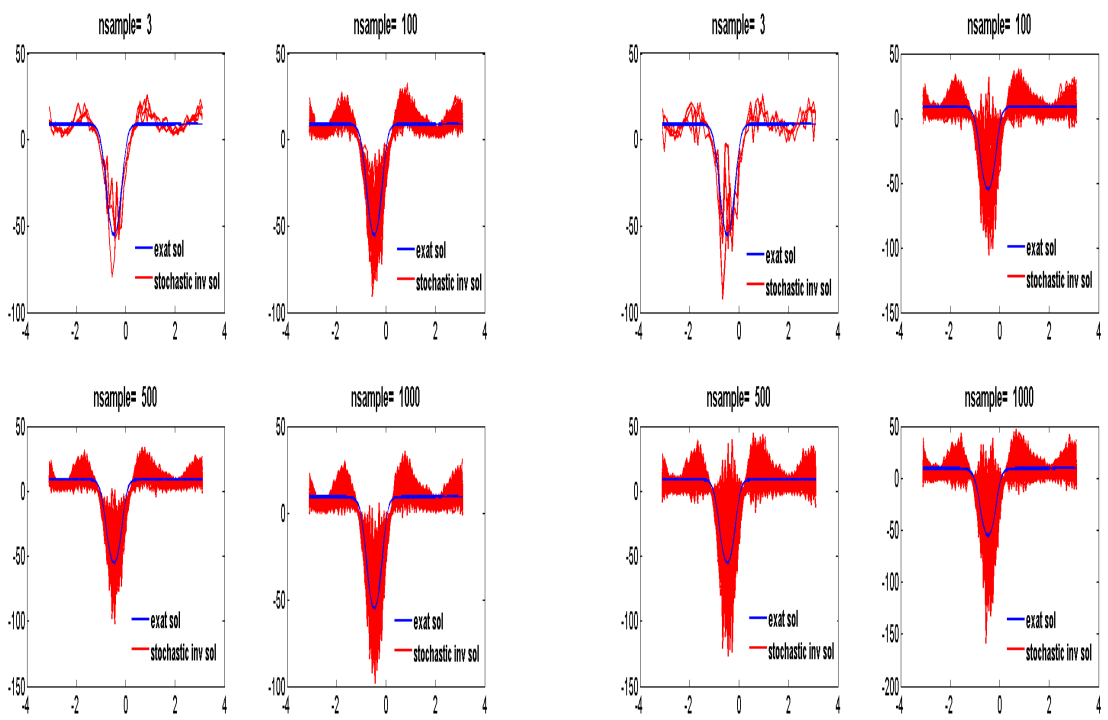

(c)

(d)

FIGURE 4. representation of the stochastic behavior of the reconstructed epicardial potential $(\tau)$ taking into account the torso data uncertainty, for different numbers of samples, each panel gather four plots showing the exact epicardial data solution (blue line) and from left to right top to bottom the simulation of 10, 100, 500, and 1000 samples of $(\tau)$ (red line). Panel (a), (respectively panel (b), panel (c) and panel (d)) shows the obtained results for $\nu=1 \%$ (respectively $\nu=10 \%, 30 \%$ and $50 \%$ ) 

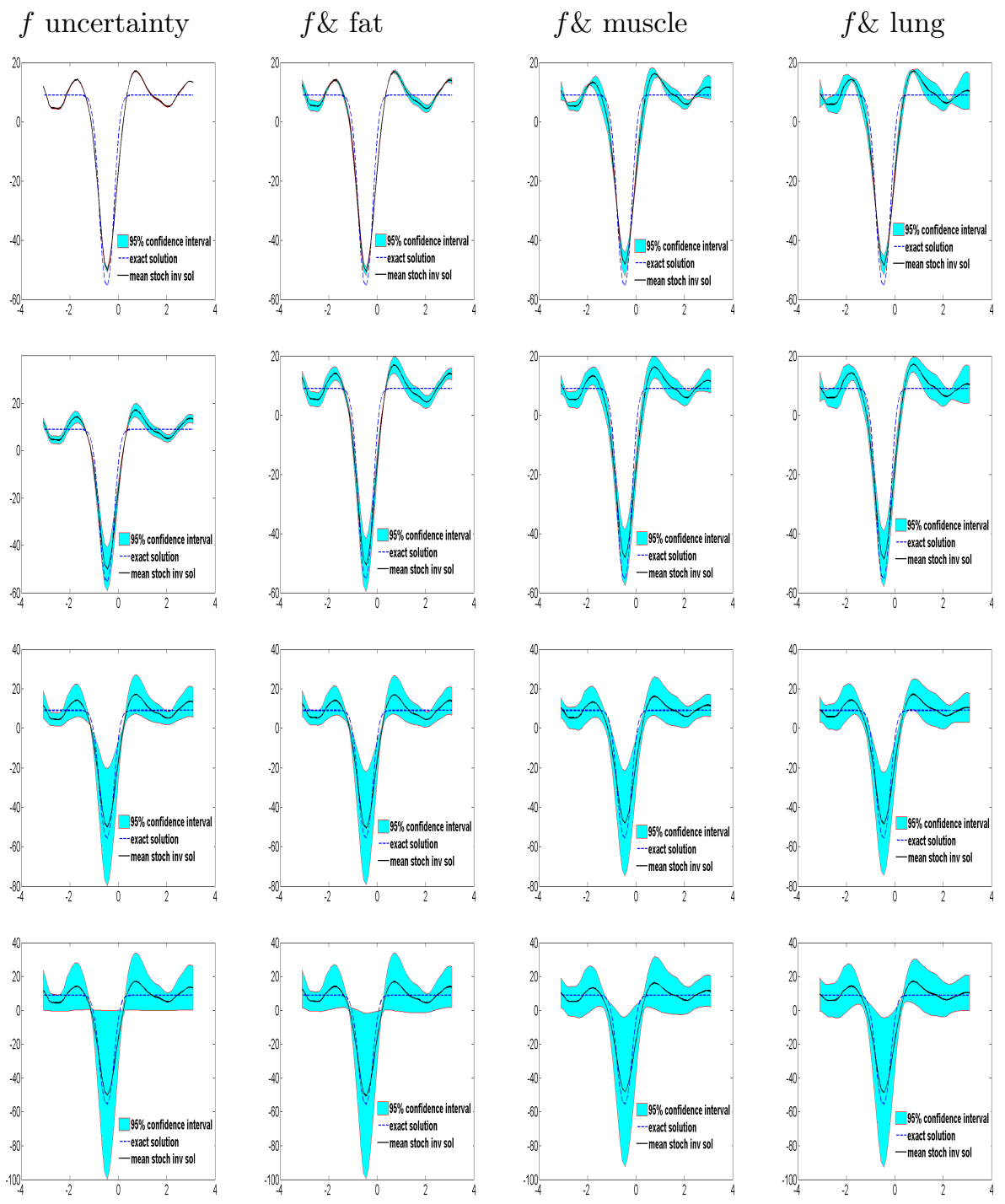

FiguRE 5. For the reconstructed stochastic field $\tau$, we show the mean value of the SFEM inverse solution (black line), exact solution (blue dashed line) and the $95 \%$ confidence interval (cyan region bounded by red lines), with respect to the case without uncertainties for different levels of $\nu=1 \%$ (respectively from top to bottom $\nu=1 \%, \nu=10 \%, \nu=30 \%, \nu=50 \%$ ), and from the left to the right we depict the case with only the torso data uncertainty (respectively torso data uncertainty with $\pm 50 \%$ of fat, cavity and lung uncertainty.

Furthermore, Figure 6 illustrates for the same four cases presented in Figure 5 the distribution deviation of the SFE inverse solution $\left(v\left(x, \xi_{0}, \xi_{1}\right)\right)$, with respect to the case without uncertainties. Similarly, from top to bottom $\nu=1 \%, 10 \%, 30 \%$, and $50 \%$. The obtained results confirm our outcomes in Figure 5 . For low values of $\nu(\nu=1 \%)$, the deviation is much more pronounced for the lung conductivities case than it is for the other organs. We also have the same pattern of the deviation distribution for high values of $\nu(\nu \geq 30 \%)$. In addition, the distributions shown 
in Figure 6 allow us to localize the most sensitive regions to uncertainties with a global vision of the uncertainty propagation.
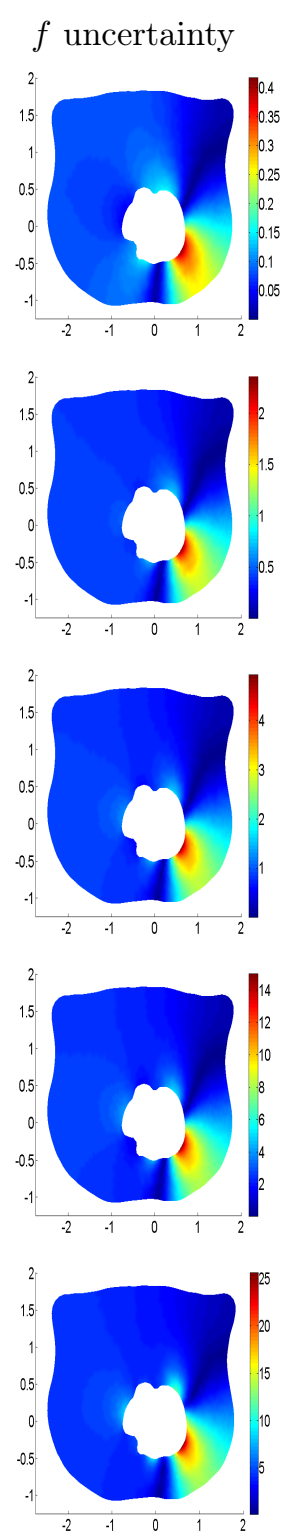

$f \&$ fat

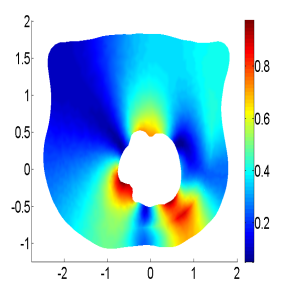

$f \&$ muscle
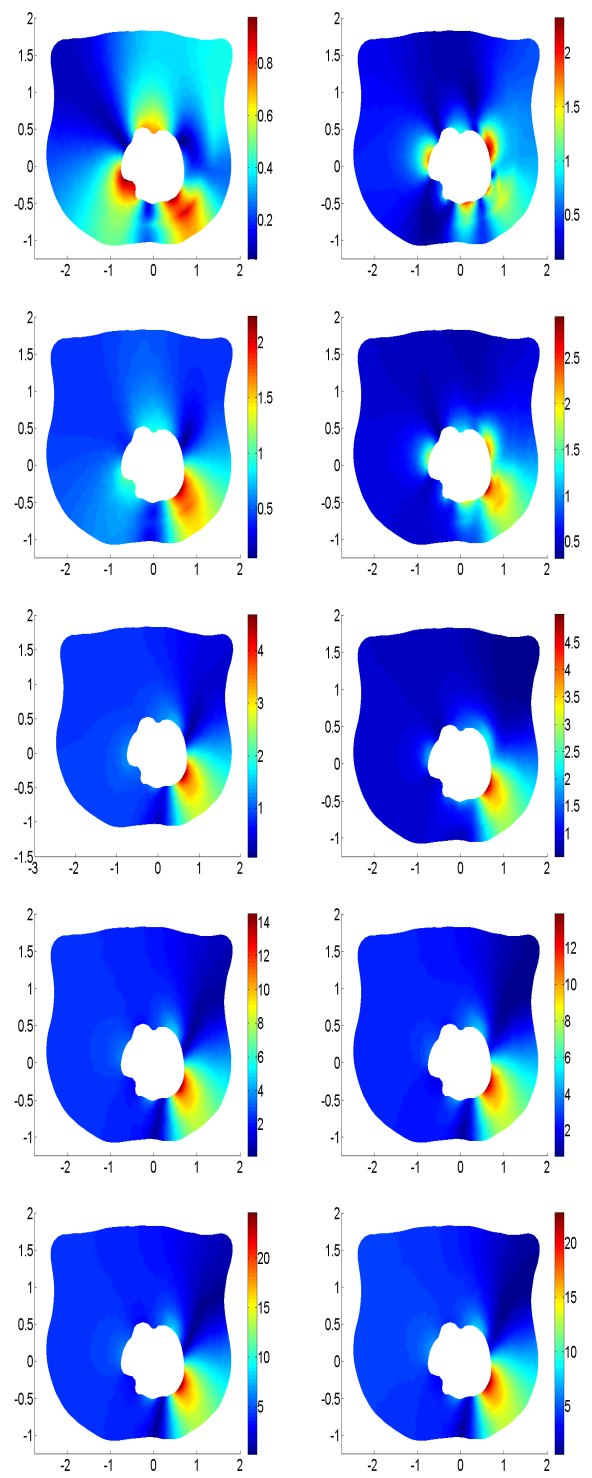

$f \&$ lung
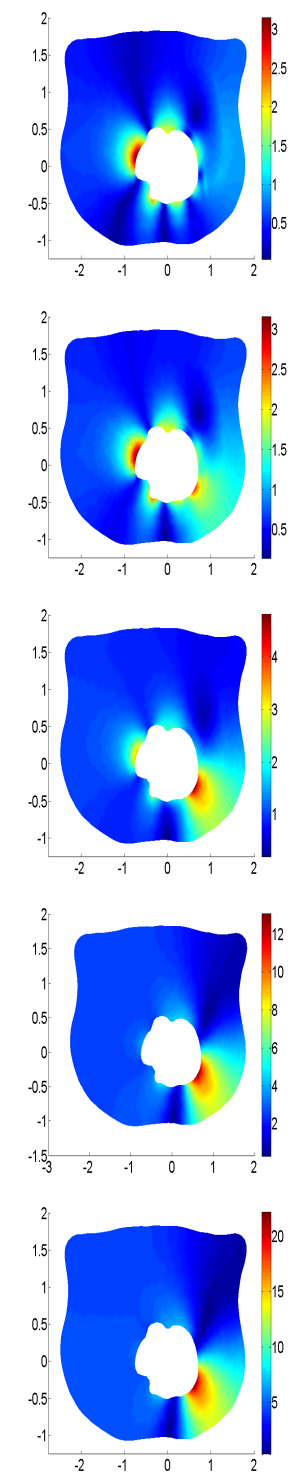

FiguRE 6 . The figure presents for different level of $\nu=1 \%$ (respectively from top the bottom $\nu=1 \%, \nu=5 \%, \nu=10 \%, \nu=30 \%, \nu=50 \%$ ), the deviation of the SFE inverse solution $\left(v\left(x, \xi_{0}, \xi_{1}\right)\right)$ with respect to the case without uncertainties. And from the left to right the panels depict the case with only the torso data uncertainty ( respectively torso data uncertainty and $\pm 50 \%$ of fat, muscle and lung uncertainties 


\section{Conclusion}

This paper presents a novel framework of the stochastic approach, allowing to combine two independent sources of uncertainty in the forward and inverse problems of ECG. The method is based on the polynomial chaos and SFE method. We formulated the inverse problem as an optimal control stochastic inverse problem in order to be able to propagate the source of uncertainty to the output of the problem (epicardial potential in this application). We proved the efficiency of this tools through various numerical simulations. Our finding is that the potential boundary data uncertainty have a strong effect on the forward and inverse problem solution, compared to the organs conductivity uncertainties. By the same token, our results permit to classify the influence of each input parameter: the lungs and the cavity conductivity uncertainties are in the same level of sensitivity. However, the uncertainty of the fat conductivity did not affect too much the inverse solution. Finally, the challenge that we propose to address in future works is to analyze the influence of the proximity in various organs to the bounding surfaces on the model accuracy, also the implementation in $3 \mathrm{D}$ of the proposed methodology.

\section{ACKNOWLEDGEMENT}

Acknowledgements. This work has been supported by EPICARD cooperative research program, funded by INRIA international laboratory LIRIMA. This study received also financial support from the French Government as part of the 'Investments of the future' program managed by the National Research Agency (ANR), Grant reference ANR-10-IAHU-04.

\section{REFERENCES}

[1] R. Aboulaich, A. B. Abda, M. Kallel, missing boundary data reconstruction via an approximate optimal control, Inverse Problems and Imaging 2(4) (2008) 411-426.

[2] R. Aboulaich, N. Fikal, E.E. Guarmah and N. Zemzemi, Stochastic finite element method for torso conductivity uncertainties quantification in electrocardiography inverse problem, Mathematical Modelling of Natural Phenomena 11(2) (2016) 1-19.

[3] S. Andrieux, T. Baranger and A.B. Abda, Solving cauchy problems by minimizing an energy-like functional, Inverse problems 22(1) (2006) 115-133.

[4] V. Asokan, B. Narayanan and N. Zabaras, Stochastic inverse heat conduction using a spectral approach, Int. J. Numer. Meth. Engng 60(7) (2004) 1-24.

[5] I.Babuska, R. Tempone and G. Zouraris, Galerkin finite element approximations of stochastic elliptic partial differential equations, SIAM Journal on Numerical Analysis 42(2) (2005) 800-825.

[6] I.Babuska, R. Tempone and G. Zouraris, Solving elliptic boundary value problems with uncertain coefficients by the finite element method: the stochastic formulation, Comput. Methods Appl. Mech. Engrg 194(2005) $1251-1294$.

[7] J. Barbara, Y. Rudy, The inverse problem in electrocardiography: A model study of the effects of geometry and conductivity parameters on the reconstruction of epicardial potentials, IEEE transactions on biomedical engineering 33(7) (1986) 667-676.

[8] M. Berveiller, Eléments finis stochastiques: approches intrusive et non intrusive pour des analyses de fiabilité, Ph.D. thesis, Université Blaise Pascal, Clermont-Ferrand (2005).

[9] M. Boulakia, C. Serge, M. Fernández, , G. Jean-Frédéric and N. Zemzemi, Mathematical modeling of electrocardiograms: a numerical study, Annals of biomedical engineering 38(3) (2010) 1071-1097.

[10] C. Bullard, A. Sebald, Monte carlo sensitivity analysis of input-output models, The Review of Economics and Statistics 70(1) (1988) 708-712.

[11] Y. Cao, M. Hussaini, T. Zang, An efficient Monte Carlo method for optimal control problems with uncertainty, Computational Optimization and Applications 26(2) (2003) 219-230.

[12] Y. Cao, Numerical solutions for optimal control problems under SPDE constraints, Tech. rep., Department of Mathematics, Florida A.M University, Tallahassee, FL (2006). 
[13] J. Ching, J. L. Beck, K. A. Porter, Bayesian state and parameter estimation of uncertain dynamical systems, Elsevier Probabilistic Engineering Mechanics 21(1) (2006) 81-96.

[14] F. Duck, Physical Properties of Tissue: A Comprehensive Reference Book, 1990.

[15] M. Eiermann, O. Ernst, E. Ullmann, Computational aspects of the stochastic finite element method Computing and Visualization in Science (1) (2007) 3-15.

[16] T. Faes, D. Van, D. Munck, R. Heethaar, The electric resistivity of human tissues (100 hz-10 mhz): a meta-analysis of review studies, Physiological measurement(4) (1999) 1-11.

[17] M. Fernández, N. Zemzemi, Decoupled time-marching schemes in computational cardiac electrophysiology and ecg numerical simulation, Mathematical biosciences 226(1) (2010) 58-75.

[18] S. Gabriel, R. Lau, C. Gabriel, The dielectric properties of biological tissues: Ii. measurements in the frequency range $10 \mathrm{hz}$ to $20 \mathrm{ghz}$, Physics in medicine and biology 41(11) (1996) 22-51.

[19] S. Geneser, R. Kirby, R. Macleod and R. Kirby, Application of stochastic finite element methods to study the sensitivity of ecg forward modeling to organ conductivity, Biomedical Engineering, IEEE Transactions 55(1) (2008) 31-40.

[20] R. Gulrajani, The forward and inverse problems of electrocardiography, EMBS Magazine 17(5) (1998) 84-101.

[21] J. Hadamard, Lectures on Cauchy's problem in linear partial differential equations, New Haven, 1923.

[22] D. Hamby, A review of techniques for parameter sensitivity analysis of environmental models, The Review of Economics and Statistics 32(2) (1994) 135-154.

[23] J. M. Holtzman, On using perturbation analysis to do sensitivity analysis: derivatives versus differences, IEEE Transactions on Automatic Control, 37(2) (1992) 243-247.

[24] L. Hou, J. Lee, H. Manouzi, Finite element approximations of stochastic optimal control problems constrained by stochastic elliptic pdes, Elsevier Journal of Mathematical Analysis and Applications 384 (2011) $87-103$.

[25] O. L. Maître, M. Reagan, H. Najm, R. Ghanem and O. Knio, A stochastic projection method for fluid flow: II. random process Elsevier Journal of Computational Physics 181(1) (2002) 9-44.

[26] A. Mugler and H.J. Starkloff, On elliptic partial differential equations with random coefficients, Babes-Bolyai Math, 56(2),(2011),473-487.

[27] A. Oosterom, G. Huiskamp, The effect of torso inhomogeneities on body surface potentials quantified using tailored geometry, Journal of electrocardiology 22(1) (1989) 53-72.

[28] C. Ramanathan, Y. Rudy, Electrocardiographic imaging: II. Effect of torso inhomogeneities on noninvasive reconstruction of epicardial potentials, electrograms and isochrones,Journal of cardiovascular electrophysiology 12(2) (2001) 241-252.

[29] E. Rosseel and G.N. Wells , Optimal control with stochastic PDE constraints and uncertain controls, Computer Methods in Applied Mechanics and Engineering, 213,(2012),152-167.

[30] H. Tiesler and R. M. Kirby and D. Xiu and Tobias Preusser, Stochastic collocation for optimal control problems with stochastic PDE constraints, SIAM J. Control optim, 50(5), (2012) 2659-2682.

[31] H. Verbeeck, R. Samson, F. Verdonck, R. Lemeur, Parameter sensitivity and uncertainty of the forest carbon flux model forug: a Monte Carlo analysis, Tree Physiology 26(1) (2006) 807-817.

[32] J. Wan, N. Zabaras, A Bayesian approach to multiscale inverse problems using the sequential Monte Carlo method, Inverse Problems, 27, Number 1027 (10),(2011) 105004 (25pp).

[33] F. Weber, D. Keller, S. Bauer, G. Seemann, C. Lorenz, O. Dossel, Predicting tissue conductivity influences on body surface potentialsan efficient approach based on principal component analysis, Biomedical Engineering IEEE Transactions 58(2) (2011) 256-273.

[34] N. Wiener, The homogeneous chaos, Am. J. Math. 60(1938),(1998) 897-936.

[35] D. Xiu, G. Karniadakis, Modeling uncertainty in flow simulations via generalized polynomial chaos, Elsevier, J.Comput.Phys. 187(1), (2003) 137-167.

[36] N. Zabaras, B. Ganapathysubramanian, A scalable framework for the solution of stochastic inverse problems using a sparse grid collocation approach, Elsevier Journal of Computational Physics 227(9) (2008) 46974735 . 